\title{
Mental disorders and conditions specifically related to stress
}

\author{
C. Barbui*, Guest Editor and M. Tansella, Editor
}

First published online 24 April 2013

Stress-related conditions are psychological reactions arising in response to traumatic events. Their prevalence in emergency situations, including armed conflicts, as well as natural and industrial disasters, is high (Tol et al., 2011). Most frequently, mental health researchers have focused on identifying rates of posttraumatic stress disorder (PTSD) and other common mental disorders, but other mental health problems are similarly relevant in these settings. In terms of age-standardized disability-adjusted life year (DALY) rates, the impact of stress-related conditions is epidemiologically relevant not only in low- and middleincome countries (LAMICs), where armed conflicts predominantly occur but also in high-income countries (HICs): considering the 25 most populated countries of the world, PTSD impact has been estimated to be the highest in Asian/Pacific countries, the USA, and Egypt, ranging from 59 DALYs in Thailand to 58 DALYs in the USA and 56 in Egypt. In Europe, estimates are high, accounting for 54 DALYs in Italy, Germany, France and the UK (World Health Organization, 2008).

Most people encounter traumatic events over a lifetime, about 20-30\% develop post-traumatic stress symptoms and less than $10 \%$ meet diagnostic criteria for PTSD (Kessler et al., 1995; Bromet et al., 1998). PTSD is an anxiety disorder that develops after exposure to any event that results in psychological trauma. This event may involve the threat of death to oneself or to someone else, or to one's own or someone else's physical, sexual or psychological integrity, overwhelming the individual's ability to cope. The lifetime prevalence of PTSD among adult Americans is 7.8\%, with women $(10.4 \%)$ more frequently affected than men (5\%) (Kessler et al., 1995; Bromet et al., 1998). The most frequently reported traumatic events by men are rape, combat and childhood neglect or physical abuse.

* Address for correspondence: Professor Corrado Barbui, Department of Public Health and Community Medicine, Section of Psychiatry, Ospedale Policlinico 'G.B. Rossi', Piazzale L.A. Scuro 10, 37134 Verona, Italy.

(Email: corrado.barbui@univr.it)
Women most frequently report instances of rape, sexual molestation, physical attack, being threatened with a weapon and childhood physical abuse.

In this issue of Epidemiology and Psychiatric Sciences Tol et al. provide a number of suggestions and recommendations for expanding the role of epidemiology in emergency situations, and for overcoming current research and clinical issues (Tol et al., 2013). One of these issues is the validity and applicability of international psychiatric diagnostic categories, in particular the diagnosis of PTSD. In this issue of Epidemiology and Psychiatric Sciences Bisson updates on recent harmonization attempts between the International Classification of Diseases (ICD) and the Diagnostic and Statistical Manual of Mental Disorders (DSM) (Bisson, 2013).

The bottom line of these two stimulating contributions can be summarized as follows. First, despite considerable efforts made in the last few decades, epidemiological studies better describing the prevalence rates of PTSD and other mental disorders in emergency settings are needed. Current studies suffer from a number of shortcomings, including the use of symptom checklists with arbitrary cut-off thresholds that may have categorized as mental disorders normal distress of individuals exposed to adverse situations. Epidemiological studies employing better measures of psychological distress and mental disorders may effectively contribute to identify risk and protective factors for the development of disorders and for the long-term consequences of exposure to traumatic events. Second, within an epidemiological framework, intervention studies should be designed to establish the feasibility and effectiveness of interventions aimed at managing psychological distress in the early phases after exposure to traumatic events. This seems particularly needed in recognition of the importance of different psychological support strategies, including preventive measures (psychological debriefing, risk targeted interventions and stepped collaborative care), psychotherapeutic interventions (cognitivebehavioural therapy, eye movement desensitization and reprocessing, and interpersonal psychotherapy), and pharmacological interventions (antidepressants, 
antipsychotics, benzodiazepines and mood stabilizers). A third issue is that epidemiological and intervention studies are meaningful if based on reliable and valid definitions. Particularly, diagnostic criteria for PTSD are a challenging issue, and current difficulties in harmonizing ICD with DSM may have negative implications. It is worth noting that modern psychiatry is still characterized by a diagnostic paradox, as ICD is routinely adopted as a reference tool when a patient is discharged from hospital, but for research purposes we switch to DSM as a reference standard.

This paradox poses a challenging issue of implementability of findings from epidemiological and intervention studies (Barbui \& Tansella, 2012). It is very difficult for the individual health care provider to interpret the existing evidence coming from clinical trials, observational studies and other sources of scientific evidence. Even if the evidence is systematically reviewed, the translation into the relevant policy and programme decisions, as well as the implementation in practice, is not straight-forward. Effective strategies, techniques, and incentives are needed to disseminate and implement current knowledge, bearing in mind that the interventions need to be adopted and integrated into practice by providers at all levels.

\section{Financial Support}

This research received no specific grant from any funding agency, commercial or not-for-profit sectors.

\section{Conflict of Interest}

None.

\section{References}

Barbui C, Tansella M (2012). Guideline implementation in mental health: current status and future goals. Epidemiology and Psychiatric Sciences 21, 227-229.

Bisson J (2013). What happened to harmonisation of the PTSD diagnosis? The divergence of ICD11 and DSM5. Epidemiology and Psychiatric Sciences 22.

Bromet E, Sonnega A, Kessler RC (1998). Risk factors for DSM-III-R posttraumatic stress disorder: findings from the National Comorbidity Survey. American Journal of Epidemiology 147, 353-361.

Kessler RC, Sonnega A, Bromet E, Hughes M, Nelson CB (1995). Posttraumatic stress disorder in the National Comorbidity Survey. Archives of General Psychiatry 52, 1048 1060.

Tol WA, Barbui C, Galappatti A, Silove D, Betancourt TS, Souza R, Golaz A, van Ommeren M (2011). Mental health and psychosocial support in humanitarian settings: linking practice and research. Lancet 378, 15811591.

Tol WA, Rees S, Silove D (2013). Broadening the scope of epidemiology in conflict-affected settings: opportunities for mental health prevention and promotion. Epidemiology and Psychiatric Sciences 22.

World Health Organization (2008). The Global Burden of Disease. World Health Organization: Geneva. 